\title{
Nonlinear terahertz dynamics of Dirac electrons in Bi thin films
}

Ikufumi Katayama, Yasuo Minami, Yusuke Arashida, Orjan Sele Handegard, Tadaaki Nagao, et al.

Ikufumi Katayama, Yasuo Minami, Yusuke Arashida, Orjan Sele Handegard, Tadaaki Nagao, Masahiro Kitajima, Jun Takeda, "Nonlinear terahertz dynamics of Dirac electrons in Bi thin films," Proc. SPIE 10756, Terahertz Emitters, Receivers, and Applications IX, 107560P (14 September 2018); doi: $10.1117 / 12.2320801$

SPIE Event: SPIE Optical Engineering + Applications, 2018, San Diego, California, United States 


\title{
Nonlinear terahertz dynamics of Dirac electrons in Bi thin films
}

\author{
Ikufumi Katayama*a, Yasuo Minami ${ }^{\text {a,b }}$, Yusuke Arashida ${ }^{\text {a }}$, \\ Orjan Sele Handegard, ${ }^{\mathrm{c},}$, Tadaaki Nagao ${ }^{\mathrm{c}, \mathrm{d}}$, Masahiro Kitajima ${ }^{\mathrm{a}, \mathrm{c}}$ and Jun Takeda ${ }^{\mathrm{a}}$ \\ ${ }^{a}$ Yokohama National University, 79-5 Tokiwadai, Hodogaya, Yokohama, JAPAN 240-8501; \\ ${ }^{\mathrm{b}}$ Tokushima University, Minami-Josanjima 2-1, Tokushima, JAPAN 770-8506; \\ ${ }^{c}$ National Institute for Materials Science (NIMS), Namiki 1-1, Tsukuba, Ibaraki 305-0044, Japan; \\ ${ }^{\mathrm{d}}$ Hokkaido University, Kita 10, Nishi 8, Kita-ku, Sapporo 060-0810, Japan
}

\begin{abstract}
By using both linear and nonlinear terahertz spectroscopy on epitaxial $\mathrm{Bi}$ and $\mathrm{Bi}_{1-x} \mathrm{Sb}_{x}$ thin films, we systematically investigated the linear and nonlinear terahertz dynamics of Dirac electrons. The linear terahertz transmittance was analyzed by the Drude model up to $50 \mathrm{THz}$, and then the plasma frequency and the damping constant were evaluated as functions of the film thickness and Sb-concentration. We found surface metallic state for Bi ultra-thin films, while semimetal to semiconductor crossover for $\mathrm{Bi}_{1-x} \mathrm{Sb}_{x}$ thin films. In the nonlinear terahertz spectroscopy, the terahertz transmittance increases with increasing the field strength, which could be assigned to the carrier acceleration along the Dirac-like band dispersion at the L point in the Brillouin zone. In addition, we observed the terahertz-induced absorption in terahertz-pump and terahertz-probe spectroscopy, which could be assigned to carrier generation due to Zener tunneling in Dirac band structure. The results demonstrate that Bi-related materials are promising candidates for future nonlinear terahertz devices.
\end{abstract}

Keywords: narrow-gap semiconductor, semimetals, terahertz, Drude model, Dirac band structure *katayama-ikufumi-bm@ynu.ac.jp; phone 81 45-339-3695; fax 81 45-339-4171;

\section{INTRODUCTION}

Bismuth (Bi) is one of the well-known materials that has been extensively studied in condensed matter physics [1]. It has a very small Fermi surface and bandgap at $L$ point of the Brillouin zone, and exhibits large magneto resistance, large diamagnetism, and quantum size effect. These extraordinary physical properties have drawn considerable interest in the physics of Bi-related materials even in the recent years [2]. In particular, large atomic number of the Bi yields strong relativistic effect such as the spin-orbit coupling, leading to characteristic electronic states such as surface metallic and prototypical topological surface states [2,3]. The semimetal to semiconductor transition is also expected by tuning physical parameters such as doping level, pressure, and sample thickness [3-6].

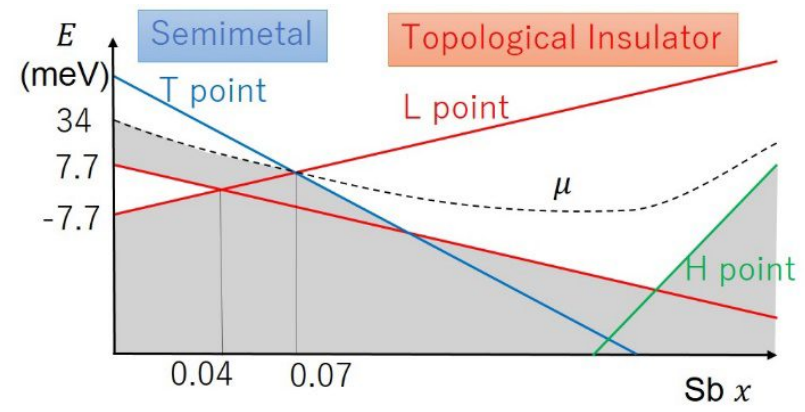

Figure 1. Schematic of the band alignment near the Fermi energy $\mu$ of $\mathrm{Bi}_{1-x} \mathrm{Sb}_{x}$. L-point electrons have a hyperbolic band dispersion, whose band gap linearly changes with $x$.

It is currently considered that these interesting physical properties originate from the peculiar band structure of Bi-related materials. Bi has both electron and hole pockets in the Brillouin zone, and the band structure near the electron pocket (L point) is non-parabolic and has a small bandgap [1]. It is known that the band structure near the L point can be

Terahertz Emitters, Receivers, and Applications IX, edited by Manijeh Razeghi,

Alexei N. Baranov, Proc. of SPIE Vol. 10756, 107560P . (c) 2018 SPIE

CCC code: $0277-786 \mathrm{X} / 18 / \$ 18 \cdot$ doi: $10.1117 / 12.2320801$ 
approximated by the Wolff Hamiltonian (anisotropic Dirac Hamiltonian), yet most of the physical properties could be well interpreted by the isotropic Wolff Hamiltonian, which is written as,

$$
\mathcal{H}=\left(\begin{array}{cc}
E_{g} / 2 & i \hbar \gamma \boldsymbol{k} \cdot \boldsymbol{\sigma} \\
-i \hbar \gamma \boldsymbol{k} \cdot \boldsymbol{\sigma} & -E_{g} / 2
\end{array}\right)
$$

Here, $\boldsymbol{\sigma}$ is the Pauli matrixes, $\boldsymbol{k}$ the wavevector, and $\gamma$ the asymptotic velocity, with an analogy to Dirac Hamiltonian [1]. The band gap energy $E_{g}$ of Bi-related materials is as small as the order of a few tens of meV, making the effective mass small, and giving rise to strong interband coupling [1]. Furthermore, the band structure can be continuously modulated by the $\mathrm{Sb}$ doping; the material changes from semimetal (Weyl semimetal, $x=0.04$ ) to topological insulators $(0.07<x$ ) as shown in Fig. 1. Thus, the electronic properties in this material system are interesting, because we can systematically investigate the band structure or the Fermi energy dependence on Dirac electrons by only tuning the Sb concentration.

In contrast to the magnetic and electrical properties, the optical properties of Bi-related materials have been less studied $[7,8]$ because the intense electromagnetic wave with the energy scale close to the small band gap energy was unavailable until recently. However, recent development of the terahertz technology enables us to access the optical nonlinearity in the wide range of material systems [9]. Here, we demonstrate that Bi-related materials show extremely large nonlinearity in the terahertz range, because of the characteristics of Dirac electrons: light effective mass and the narrow band gap [10]. In the following sections, we will describe how high-crystallinity Bi-related thin films were fabricated and characterized [11], and what exotic electronic responses to the terahertz field were observed.

\section{EXPERIMENT}

\subsection{Fabrication of Bi-related thin films}

We deposited epitaxial thin films of $\mathrm{Bi}$ and $\mathrm{Bi}_{1-x} \mathrm{Sb}_{x}$ alloys on a silicon (7X7) reconstructed surface using a homemade molecular beam epitaxy (MBE) chamber [11]. The base pressure of the chamber was kept below $10^{-7} \mathrm{~Pa}$, which ensured low impurity concentration in the sample. We simultaneously evaporated both $\mathrm{Bi}$ and $\mathrm{Sb}$ from two Knudsen-cells with the controlled evaporation rates. The total evaporation rate was kept below $0.02 \mathrm{~nm} / \mathrm{min}$. to obtain high-crystalline films. The film thickness was monitored by a conventional crystal oscillator, and the $\mathrm{Sb}$ concentration was cross checked by using X-ray fluorescence and X-ray photoelectron spectroscopy. We confirmed the crystallinity of the films by using reflection high-energy electron diffraction (RHEED). The samples were then post-annealed at $350 \mathrm{~K}$.

Figure 2(a) shows a typical RHEED pattern for the silicon (7X7) reconstructed surface used for the substrate, indicating the high flatness of the reconstructed surface. The typical RHEED pattern for the deposited thin film (Bi film with $40 \mathrm{~nm}$ thickness) is shown in Fig. 2(b). The distinct diffraction spots in the picture illustrate the high crystallinity of the deposited thin films, which agrees well with the previous results reported in the literature [3].
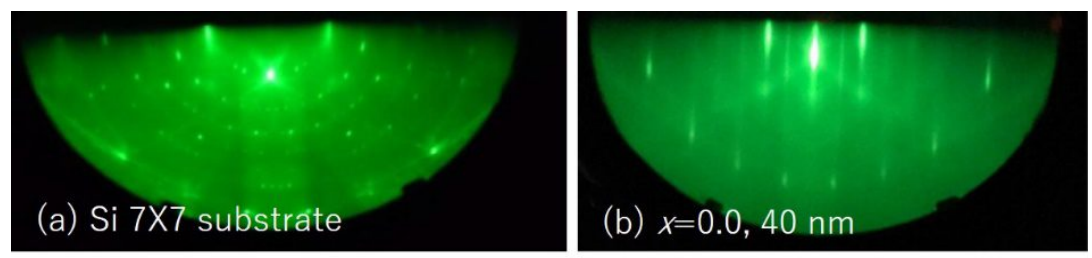

Figure 2. RHEED patterns of the $\mathrm{Si}$ substrate and $\mathrm{Bi}_{1-x} \mathrm{Sb}_{x}$ thin film $(x=0)$. Energy of the incident electrons was $15 \mathrm{keV}$.

\subsection{Linear terahertz spectroscopy}

For understanding the carrier dynamics in $\mathrm{Bi}_{1-x} \mathrm{Sb}_{x}$ thin films, we first examined the terahertz transmittance using a homemade broadband terahertz time-domain spectrometer that covers up to $10 \mathrm{THz}$. The broadband terahertz wave was generated from a photo-conductive antenna excited via a 15-fs Ti:sapphire oscillator with an output power of $400 \mathrm{~mW}$ and a repetition rate of $80 \mathrm{MHz}$ in the reflection configuration, whose geometry avoids the terahertz absorption from the substrate [12]. The transmittance for the higher frequency region up to $50 \mathrm{THz}$ was measured by a conventional Fourier transform infrared (FTIR) spectrometer. The transmittance was defined by the ratio of the terahertz spectrum transmitted through the $\mathrm{Bi}_{1-x} \mathrm{Sb}_{x}$ thin films to that through the Si substrate.

The observed broadband terahertz transmittance spectra for different film thicknesses and Sb concentrations are shown in Figs. 3(a) and 3(b). To characterize free carriers for each $\mathrm{Bi}_{1-x} \mathrm{Sb}_{x}$ thin film in broadband terahertz frequency regions, 
we calculated the whole spectrum using the Drude model combined with the Tinkham equation as $\tilde{T} / T_{0}=(1-$ $i \varepsilon \omega d /(n+1) c)^{-1}[6]$. Here, $d$ is the film thickness, $n$ the refractive index of the substrate, and $c$ the speed of light. Then, the complex dielectric dispersion due to the free carriers is given by the Drude model as,

$$
\varepsilon=\varepsilon_{\infty}-\frac{\omega_{p}^{2}}{\omega(\omega+i \Gamma)}
$$

where $\omega_{p}$ is the plasma frequency, $\Gamma$ the damping constant, and $\varepsilon_{\infty}$ the effective high-frequency dielectric constant. Eventually, we could obtain the plasma frequency and damping constant for each sample as shown in Figs. 3(c-f). The plasma frequency and damping constant increase with decreasing the film thickness, indicating the increase of the contribution from the surface metallic state. On the other hand, the plasma frequency decreases and the damping constant increases with increasing the Sb concentration up to $x=0.07$, which is consistent with the semimetal to semiconductor transition. Note that thermally excited carriers might have a significant contribution even in the case of semiconducting samples $(x>0.07)$, reflecting the characteristic of the narrow bandgap in $\mathrm{Bi}_{1-x} \mathrm{Sb}_{x}$ samples. The results indicate that the broadband terahertz spectroscopy is suitable for characterizing the carrier properties in Bi-related materials.
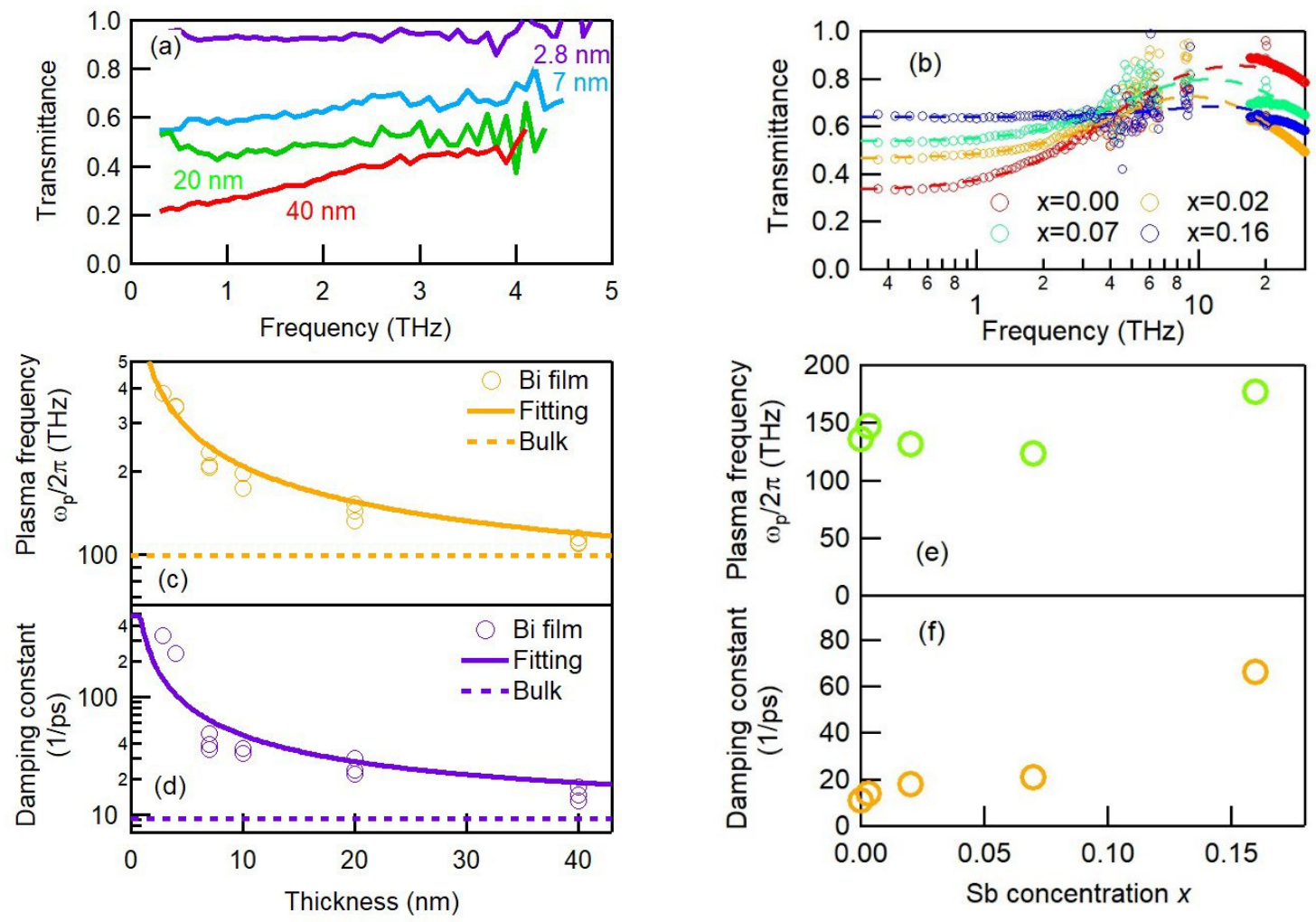

Figure 3. (a) Terahertz transmittance spectra of $\mathrm{Bi}$ thin films with different film thicknesses. (b) Terahertz transmittance spectra of $\mathrm{Bi}_{1-x} \mathrm{Sb}_{x}$ thin films with a thickness of $40 \mathrm{~nm}$. Dashed lines are the fittings using the Drude model. (c), (d) Thickness dependence of the plasma frequency and damping constant calculated from the data in (a) with the Drude model. Solid lines are the fittings considering the contribution from both bulk and surface carriers [6], while the dashed lines indicate the values of bulk $\mathrm{Bi}$. (e), (f) The Sb-concentration dependence of the plasma frequency and damping constant calculated from the data in (b) with the Drude model.

\subsection{Nonlinear terahertz spectroscopy}

To investigate the nonlinear carrier behaviors of Bi-related materials, we used the experimental setup similar to that previously reported [13]. A Ti:sapphire regenerative amplifier with an output power of $2 \mathrm{~mJ}$, a repetition rate of $1 \mathrm{kHz}$, and a center wavelength of $800 \mathrm{~nm}$ was used as a light source. The laser output was divided into three beams. These were used for generating the terahertz pump and probe beams, and for gating the electrooptic (EO) sampling to measure the electric field of transmitted terahertz waveforms. For generating the terahertz pump and probe beams, we shined the laser pulses with a given delay time on a $\mathrm{LiNbO}_{3}$ single crystalline prism with a pulse-front tilt configuration to satisfy 
the non-collinear phase matching [14]. The electric fields for the pump and probe beams were detected with the EO sampling method with a 400- $\mu \mathrm{m} \mathrm{GaP} \mathrm{(110)} \mathrm{single} \mathrm{crystal.} \mathrm{The} \mathrm{maximum} \mathrm{electric} \mathrm{field} \mathrm{strength} \mathrm{for} \mathrm{the} \mathrm{pump} \mathrm{terahertz}$ pulse was $80 \mathrm{kV} / \mathrm{cm}$, while the typical electric field for the probe terahertz pulse was $4 \mathrm{kV} / \mathrm{cm}$ at the sample position.

We summarize the terahertz-pump-induced transmittance change of $\mathrm{Bi}_{1-x} \mathrm{Sb}_{x}$ thin films $(x=0,0.16)$ with different electric field strengths. As shown in Fig. 4, in which the terahertz transmittance change increases with increasing the field strength in the case of semimetal samples (typically, $x=0$ ). On the contrary, the transmittance slightly decreases with increasing the field strength in the case of semiconducting samples $(x=0.16)$. The results demonstrate that two processes may contribute to the observed carrier nonlinearity in Bi-related thin films.

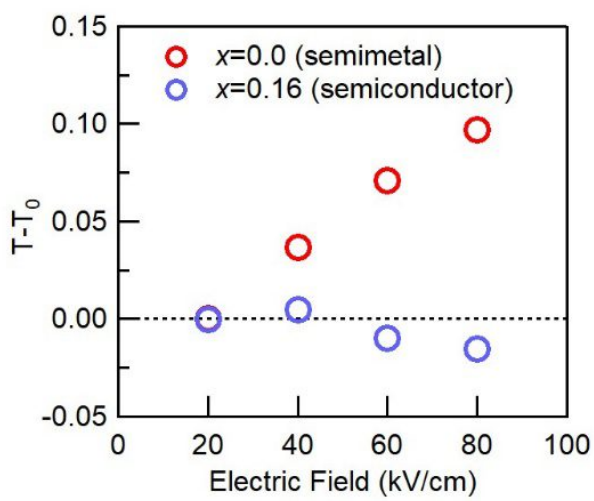

Figure 4. Terahertz-pump-induced transmittance change as a function of the terahertz electric field strength. The difference between the data at $20 \mathrm{kV} / \mathrm{cm}$ and that at given electric fields are plotted.

To elucidate the origins of the terahertz-pump-induced nonlinearity, we performed the terahertz-pump and terahertzprobe spectroscopy for $\mathrm{Bi}_{1-x} \mathrm{Sb}_{x}$ thin films. Figure 5 shows the obtained terahertz induced transmittance change as a function of the pump-probe delay time for $x=0$ and $x=0.16$ samples. At the time origin, the transmittance abruptly increases in the case of semimetal Bi thin film, and then, the terahertz-pump-induced absorption with a relaxation time of $\sim 6 \mathrm{ps}$ appears for both semimetal and semiconducting thin films. The induced absorption could be related to the carrier generation process. Note that the second peak observed around $\sim 9 \mathrm{ps}$ is due to the reflection either from the substrate or an EO crystal.

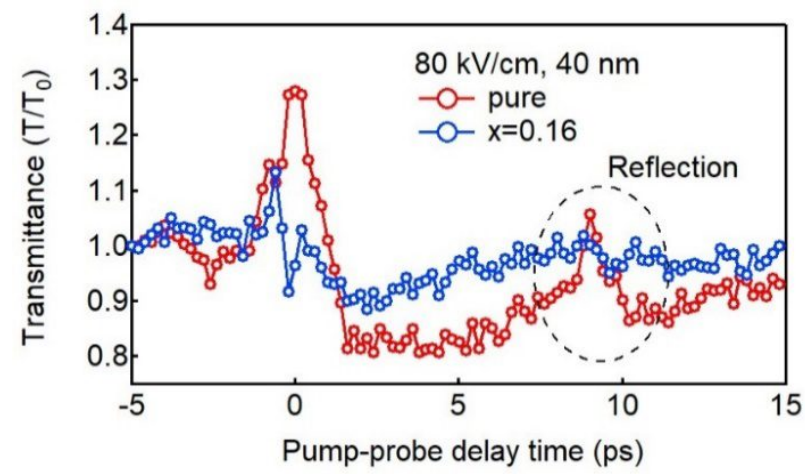

Figure 5. Transmittance change of the probe terahertz pulse as a function of the pump-probe delay time induced by the pump terahertz pulse with peak electric field strength of $80 \mathrm{kV} / \mathrm{cm}$. Ratio of the peak electric field strength of the probe under pump irradiation to that without it was plotted. The thickness of the films were $40 \mathrm{~nm}$. 


\section{DISCUSSIONS}

\subsection{Terahertz field acceleration}

To understand the nonlinear terahertz behaviors of the Bi-related thin films, we consider the model Hamiltonian with the isotropic hyperbolic band structure as $E= \pm \sqrt{E_{g}^{2} / 4+\gamma^{2} \hbar^{2} k^{2}}$ [1]. The band parameters for pure Bi at room temperature are $\mathrm{Eg}=15.4 \mathrm{meV}$ and asymptotic velocity $c=343 \mathrm{~m} / \mathrm{s}$ [10]. Then the current induced by the carriers in the conduction band should be written as

$$
J=-e N v=-e N \frac{\partial E}{\partial p} .
$$

Here, $-e$ is the charge of an electron, $N$ the carrier density of the film, $v$ the velocity of the electrons, and $p$ the momentum. Because of the non-parabolic band structure, $v$ depends on the energy (momentum) of the electrons as in the case of special relativity. If the conduction electrons are accelerated to higher energy through terahertz electric fields, the effective mass increases and the induced current decreases, resulting in the increase of the terahertz transmission, as illustrated in Fig. 4.

This nonlinear current behavior should be included in Maxwell's equations. For simplicity, we assume that the sample thickness is thin enough compared to the wavelength of the terahertz wave. In this case, the terahertz electric field applied on the thin film could be approximated by the following formula [10].

$$
E_{\text {film }}=\frac{2 E_{i n}-Z_{0} J d}{n+1}
$$

Here, $E_{\text {in }}$ is the incident terahertz electric field, and $Z_{0}$ the vacuum impedance. Then, the equation of motion for the conduction electrons are

$$
\frac{\partial p}{\partial t}=-e E_{f i l m}-\Gamma p
$$

By solving these equation in the time domain, the terahertz-induced nonlinear transmittance could be numerically obtained by taking the ratio of the spectrum of $E_{f i l m}$ to that of $E_{\text {in }}$, as shown by the dashed curve in Fig. 6 . The good agreement between the experimental and theoretical results demonstrates that the nonlinear increase of the transmittance observed in Fig. 4 and 6 is due to the acceleration effect of the conduction electrons in the Dirac band [10]. According to the calculation, the effective mass increases by approximately 2.4 times as heavy as the effective rest mass of the Dirac electrons. These results show that the Dirac band structure plays a key role for the observed large terahertz nonlinearity in conductive semimetallic Bi-related systems.

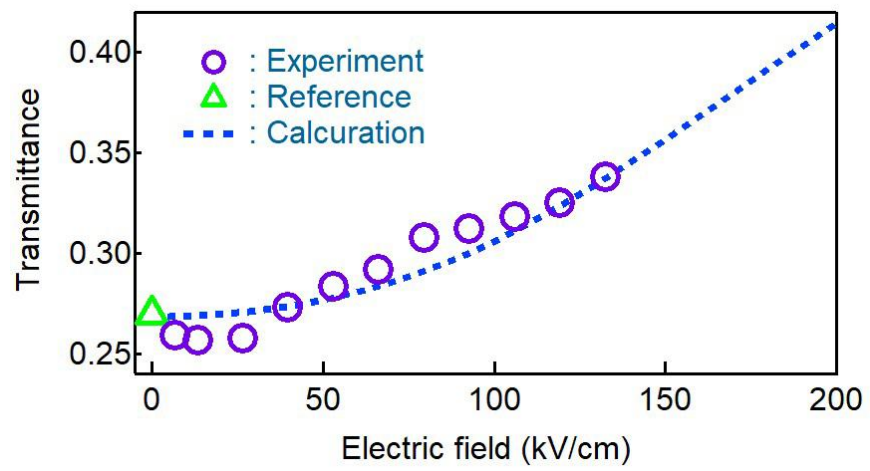

Figure 6. Transmittance change as a function of applied electric field of the terahertz pulse. The dashed line indicates the calculation written in the text. Green triangle represents the data taken from the reference [8].

\subsection{Field induced carrier generation}

Finally, we also consider the carrier generation processes observed in the terahertz-pump and terahertz-probe spectroscopy. The origin of the terahertz-induced absorption (or transmission decrease) can be assigned to either the terahertz-induced decrease of damping constant or the terahertz-induced increase of the plasma frequency. However, the decrease of the damping constant is unfavorable because the electron temperature should increase after the intense pump 
terahertz irradiation, resulting in the increase of the damping constant. Thus, the increase of the plasma frequency may contribute to the observed terahertz-induced absorption, which is caused by the increase of the carrier density. There are several possible mechanisms for the increase of the carrier density under the applied terahertz field: the multi-photon excitation, field-induced tunneling, and impact ionization [15]. To distinguish these carrier generation processes, Keydish parameter could be generally used. If this parameter is smaller than 1, then the tunneling ionization dominates the nonlinear process, whereas the multiphoton ionization dominates when it is larger than 1. By calculating the Keydish parameter under our experimental condition, we obtain the value of 0.03 , indicating that the tunneling ionization would be the main mechanism for the carrier generation. We will need to calculate the tunneling probability to compare with the observed experimental results for further understanding the nonlinear carrier generation process in $\mathrm{Bi}_{1-x} \mathrm{Sb}_{x}$ Dirac electron systems.

\section{CONCLUSIONS}

In conclusion, we fabricated $\mathrm{Bi}_{1-x} \mathrm{Sb}_{x}$ thin films on a $\mathrm{Si} 7 \mathrm{X} 7$ reconstructed surfaces, and investigated their terahertzinduced nonlinear carrier dynamics. The observed terahertz-induced transmittance was analyzed by the Drude model, and the carrier dynamics in bulk and the surface states were separately obtained. By utilizing the nonlinear terahertz spectroscopy, we observed the increase of the terahertz transmittance with increasing the electric field strength in semimetallic $\mathrm{Bi}_{1-x} \mathrm{Sb}_{x}$ thin films, which could be understood by the strong acceleration along the non-parabolic Dirac band structure. Using the terahertz-pump and terahertz-probe spectroscopy, we observed the terahertz-induced transmission decrease due to the carrier generation process. The results demonstrate that Bi-related thin films are suitable materials for observing the strong nonlinearity at the terahertz region even at room temperature.

\section{ACKNOWLEDGEMENT}

Authors thank Lie-Wei Nien and Naoki Furuhata of NIMS for their support for film deposition, Koichiro Yokota, Kotaro Araki, Hiroki Kawakami, and Toshio Hagiwara of Yokohama National University for conducting the terahertz experiments.

\section{REFERENCES}

[1] Y. Fuseya, M. Ogata, and H. Fukuyama, "Transport Properties and Diamagnetism of Dirac Electrons in Bismuth,” J. Phys. Soc. Jpn. 84, 012001 (2015).

[2] D. Hsieh, D. Qian, L. Wray, Y. Xia, Y.S. Hor, R.J. Cava, M.Z. Hasan, “A topological Dirac insulator in a quantum spin Hall phase," Nature 452, 970 (2008).

[3] T. Hirahara, Y. Sakamoto, Y. Saisyu, H. Miyazaki, S. Kimura, T. Okuda, I. Matsuda, S. Murakami, S. Hasegawa, "Topological metal at the surface of an ultrathin $\mathrm{Bi}_{1-\mathrm{x}} \mathrm{Sb}_{\mathrm{x}}$ alloy film," Phys. Rev. B 81, 165422 (2011).

[4] T. Hirahara, I. Matsuda, S. Yamazaki, N. Miyata, S. Hasegawa, T. Nagao, "Large surface-state conductivity in ultrathin Bi films," Appl. Phys. Lett. 91, 202106 (2007).

[5] S. Ito et al., "Proving Nontrivial Topology of Pure Bismuth by Quantum Confinement," Phys. Rev. Lett. 117, 236402 (2016).

[6] K. Yokota et al., "Surface metallic states in ultrathin $\mathrm{Bi}(001)$ films studied with terahertz time-domain spectroscopy," Appl. Phys. Lett. 100, 251605 (2012).

[7] W. S. Boyle, A. D. Brailsford, "Far Infrared Studies of Bismuth," Phys. Rev. 120, 1943 (1960).

[8] R. Tediosi, N. P. Armitage, E. Giannini, D. van der Marel, "Charge carrier interaction with a purely electronic collective mode: plasmarons and the infrared response of elemental bismuth," Phys. Rev. Lett. 99, 016406 (2007).

[9] T. Kampfrath, K. Tanaka, K. A. Nelson, "Resonant and nonresonant control over matter and light by intense terahertz transients," Nat. Photon. 7, 680 (2013).

[10] Y. Minami, K. Araki, T.D. Dao, T. Nagao, M. Kitajima, J. Takeda, and I. Katayama, "Terahertz-induced acceleration of massive Dirac electrons in semimetal bismuth," Sci. Rep. 5, 15870 (2015). 
[11] T. Nagao et al., "Nanofilm allotrope and phase transformation of ultrathin Bi film on Si(111)-7x7," Phys. Rev. Lett. 93, 105501 (2004).

[12] I. Katayama, H. Shimosato, D. S. Rana, I. Kawayama, M. Tonouchi, M. Ashida, "Hardening of the ferroelectric soft mode in $\mathrm{SrTiO}_{3}$ thin films," Appl. Phys. Lett. 93, 132903 (2008).

[13] J. Hebling et al., "Observation of nonequilibrium carrier distribution in $\mathrm{Ge}, \mathrm{Si}$, and $\mathrm{GaAs}$ by terahertz pumpterahertz probe measurements", Phys. Rev. B 81, 035201 (2010).

[14]H. Hirori, A. Doi, F. Blanchard, and K. Tanaka, "Single-cycle terahertz pulses with amplitudes exceeding 1 $\mathrm{MV} / \mathrm{cm}$ generated by optical rectification in $\mathrm{LiNbO}_{3}$," Appl. Phys. Lett. 98, 091106 (2011).

[15] K. Tanaka, H. Hirori, and M. Nagai, "THz Nonlinear Spectroscopy of Solids," IEEE Trans. Terahertz Sci. Tech. 1, 301-312 (2011). 